\title{
Repetition benefit in mental rotation is independent of stimulus repetition
}

\author{
Qun Wan • Chuansheng Chen • Chenyang Wu • \\ Xiuying Qian
}

Published online: 28 December 2010

(C) The Author(s) 2010 This article is published with open access at Springerlink.com

\begin{abstract}
In this study, we investigated whether there is a repetition benefit in mental rotation that is independent of stimulus repetition (i.e., due to increased efficiency in postencoding processing). Three experiments were conducted, in which different conditions of stimulus repetition (different letters on consecutive trials in Experiment 1, letters of different orientations on consecutive trials in Experiment 2, and priming of rotation direction in Experiment 3) were used, and the extent of repetition of rotation direction between two consecutive trials was manipulated. The results of all three experiments showed clear evidence of a repetition benefit without repeating the stimulus, suggesting that this effect is independent of stimulus repetition and lending support to the notion of increased efficiency in mental rotation as a result of repeated rotation direction per se.
\end{abstract}

Keywords Mental rotation - Repetition benefit

Previous research has consistently demonstrated a repetition effect; that is, faster responses to a stimulus when the stimulus-response pairing is repeated than when a new pairing is involved (Schacter, Chiu, \& Ochsner 1993). There are several possible mechanisms for the repetition benefit. First, repetition of the same stimulus can lead to

Q. Wan $\cdot$ C. Wu $\cdot$ X. Qian $(\bowtie)$

Department of Psychology and Behavioral Sciences,

Zhejiang University,

Hangzhou 310028, China

e-mail: xqian@zju.edu.cn

C. Chen

Department of Psychology and Social Behavior, University of California,

Irvine, USA encoding efficiency of the stimulus because it was processed on the preceding trial. With this mechanism, the repetition benefit should be limited to identical stimuli (Wiggs \& Martin, 1998). For example, Koriat and Norman $(1988,1989)$ found that the repetition effect in mental rotation was, indeed, limited to the same-character-sameformat condition and did not extend to different characters or different formats. Another possibility is that the retrieval of a response is more efficient because the same action was performed on the preceding trial (Dobbins, Schnyer, Verfaellie, \& Schacter 2004; Pashler \& Baylis, 1991). A third plausible mechanism involves a general response selection. In a speeded-choice task, a stimulus is classified into a category depending on its task demand characteristics, and the category is mapped onto a response. For example, if a task requests the subject to press the right key for a red stimulus and to press the left key for a green stimulus, the subject needs to determine whether the stimulus is red or green (whether it is a square or a circle is not relevant), and then press the corresponding key. In this kind of task, the response selection may be specific not to the particular stimulus but, rather, to its category, because different stimuli (e.g., red square and red circle) can be classified into the same category (i.e., red stimuli). When the pairing of stimulus category and response is strengthened in a trial, it can be transferred to the subsequent trial (Meiran, 2000; Meiran \& Gotler, 2001; Steinhauser \& Hübner, 2006). According to this perspective, the repetition benefit should transfer to different stimuli as long as they belong to the same category as the stimulus presented on the preceding trial.

All three possibilities mentioned above emphasize stimulus identity, or at least stimulus similarity. Is there a repetition effect that is independent of stimulus repetition? In other words, even if the stimuli are quite different, can a 
repetition effect be elicited if efficiency can be enhanced at the level of mental processes after the encoding of stimuli?

Previous research has not addressed this possibility, because most research on the repetition effect has used speeded-choice tasks. In such tasks, there is no separation between the efficiency of stimulus encoding and that of post-encoding processes. For instance, in the classic task-switching paradigm (Monsell, 2003), task switching is obviously accompanied by a switch of the postencoding processes. Therefore, the switching cost (or repetition benefit) - namely, impaired performance for switched trials, as compared with repeated trials (Monsell, 2003) - can be attributed to either cue switching (Altmann \& Gray, 2008; Mayr \& Kliegl, 2003) or stimulus-response binding (Mayr \& Kliegl, 2003; Monsell, Yeung, \& Azuma 2000; Philipp, Jolicœur, Falkenstein, \& Koch 2007; Steinhauser \& Hübner, 2006). Consequently, it is not clear whether any of the repetition benefit could have been derived from postencoding processes.

One way to separate the contributions of stimulus repetition from increased efficiency in postencoding processes is to use a mental rotation task. Such tasks can yield indices of overall performance (i.e., the response times [RTs] and error rates), as well as the efficiency of a particular postencoding process - mental rotation. The latter can be indexed by the slope of the RT function in terms of the orientation angle $(\mathrm{OA})$ of the stimulus. In the present study, we specifically examined the effect of repeating or switching the direction of 2-D mental rotation (on a plane) (Heil, Bajrić, Rösler, \& Hennighausen 1997; Wexler, Kosslyn, \& Berthoz 1998; Wohlschläger \& Wohlschläger, 1998). We examined whether the rate of mental rotation would increase when the direction was repeated as compared to when it was switched. If the repetition of rotation direction led to an increase in the rate of mental rotation even when the successive stimuli were different, that would provide evidence of an increased efficiency in mental rotation that was independent of stimulus repetition. Furthermore, the size of the repetition effect was expected to be related to the absolute OA (AOA) of the current stimulus. With increasing AOA, more rotation would be involved, leading to a larger repetition effect (i.e., an increased slope of the RT function).

Although several studies (Koriat \& Norman, 1984, 1988; Robertson, Palmer, \& Gomez 1987) have already reported repetition effects with mental rotation tasks, those studies were designed to test hypotheses about the nature of mental rotation (e.g., frame vs. image rotation) and, hence, were not able to address our research question. Nevertheless, their results are relevant. For example, Table 1 for Experiment 1 in Koriat and Norman's study (1984) showed mean RT by rotation direction and stimulus repetition. The trend of the data appeared to reveal that the RT was smaller when the rotation direction was repeated than when it was switched and that the decrease was larger when the angular
Table 1 Orientation angle (OA) and response times (RTs): Experiments 1 and 2

\begin{tabular}{lll}
\hline OA & Exp. 1 RT (SE) & Exp. 2 RT (SE) \\
\hline $140^{\circ}$ & $598.21(12.36)$ & $642.16(14.96)$ \\
$70^{\circ}$ & $478.72(9.85)$ & $535.15(14.92)$ \\
$0^{\circ}$ & $449.87(10.08)$ & $488.38(12.69)$ \\
$+70^{\circ}$ & $487.22(11.82)$ & $546.72(15.20)$ \\
$+140^{\circ}$ & $623.61(12.89)$ & $675.31(22.61)$ \\
\hline
\end{tabular}

difference between the stimulus and the upright position was larger. These results appeared to be consistent with our hypothesis, but they were neither statistically tested nor separated by types of stimuli (the same or different stimuli across successive trials). Without the same-letter condition being separated from the different-letter condition, it was not clear whether the rotation repetition effect was driven by repeated letters only, different letters only, or both conditions. In their later two articles (Koriat \& Norman, 1988, 1989), types of stimuli were separated, but rotation repetition was not examined. Similarly, Robertson, Palmer, and Gomez (1987) showed similar data on general repetition effects without separating them according to the letter repetition and letter-switching conditions or showing the RT changes by the levels of AOA of the stimulus.

In sum, there is some secondary evidence in support of our hypothesis, but a rigorous test is needed to control for stimulus repetition and to statistically test the magnitude of the repetition effect based on postencoding mental processes. In the present study, three experiments were designed to accomplish that aim. In Experiment 1, six asymmetrical English letters (three in uppercase $[\mathrm{R}, \mathrm{L}, \mathrm{F}]$ and three in lowercase $[e, h, t]$ ) were used to examine the repetition effect of different stimuli. To control for the consistent right-facing letters (which would affect natural rotation direction) used in Experiment 1, Experiment 2 balanced the design by using one right-facing English letter (G), one left-facing English letter $(\mathrm{J})$, one right-facing Chinese character (亡), and one left-facing Chinese character (刀). In Experiment 3, we primed subjects to perform mental rotation in a given direction and examined its effect on the subsequent mental rotation task. Because we aimed to examine the repetition effect that is independent of stimulus repetition, we included only conditions with no stimulus repetition.

\section{Experiment 1}

Method

Subjects Twelve undergraduate students (5 male and 7 female) at Zhejiang University participated in the experi- 
ment. Each subject was paid 20 yuan. This study was approved by the Department of Psychology and Behavioral Sciences at Zhejiang University.

Stimuli and apparatus The stimuli were six asymmetrical alphabetic characters (e, h, t, R, L, and F). They were presented in either normal or mirror-reversed form at one of five OAs: $0^{\circ}, \pm 70^{\circ}$, or $\pm 140^{\circ}$ from the upright position (+/- indicate clockwise/counterclockwise rotation). Each stimulus subtended approximately $100 \times 100$ pixels and was presented in green color against a black background on a 14-in. LCD with a resolution of $1,024 \times$ 768 pixels

Procedure and design Subjects were instructed to identify whether a stimulus was normal or backward (mirror reversed), regardless of its orientation. To prevent interference from making key choices (Ilan \& Miller, 1994), subjects were asked to press "B" only for a normal stimulus as accurately and quickly as possible within $1,000 \mathrm{~ms}$. It should be noted that this go/no-go paradigm (i.e., responding only to normal stimuli) has been found to show a mental rotation similar to that for the classic two-response paradigm (Heil, Rauch, \& Hennighausen 1998). A chinsupporter was used to help subjects keep their heads upright during the experiment.

There were four blocks of 401 trials each. Following the randomly selected first trial, subjects were presented 400 trials in a pseudorandom order. These trials represented four presentations of the following 100 trials: 2 (normal or mirror-reversed stimulus on the preceding trial $) \times 5(\mathrm{OA}$ of the preceding stimulus: $0^{\circ}, \pm 70^{\circ}$, or $\pm 140^{\circ}$ ) $\times 2$ (normal or mirror-reversed stimulus on the current trial) $\times 5$ (OA of the current stimulus: $0^{\circ}, \pm 70^{\circ}$, or $\pm 140^{\circ}$ ). The first block was treated as practice and, thus, was excluded from analysis. Included in the analyses were error rates for all 1,203 trials (three blocks of 401 trials), but RT data were available only for about half of the trials because, as was mentioned above, subjects were asked to respond only to normal letters, not mirror-reversed letters.

Each trial started with a fixation of $500 \mathrm{~ms}$, followed by a 200-ms blank and then by the stimulus. The stimulus stayed on the screen until the subject responded or $1,000 \mathrm{~ms}$ had lapsed. There was a 500-ms blank before the next trial started (see Fig. 1). Stimuli used for successive trials alternated between upper and lowercase letters to ensure that all the successive trials had different stimuli.

Results and discussion

We first ran a one-way repeated measures ANOVA to test the differences in mean RTs by OA. There was a significant
A: Experiment 1

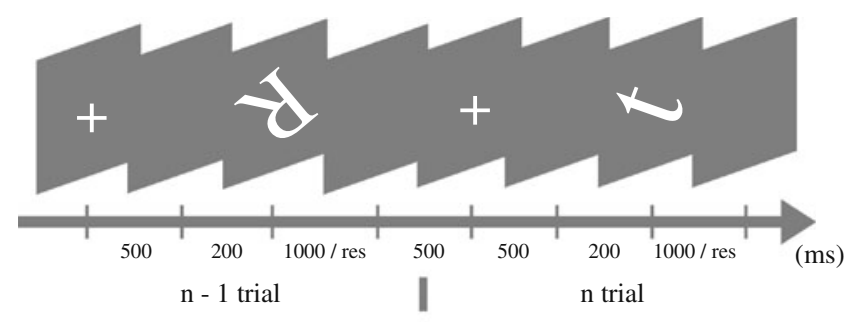

B: Experiment 2

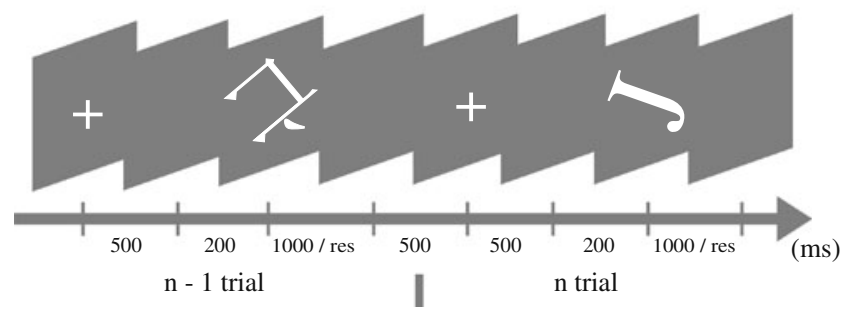

Fig. 1 Schematic depictions of the time course of two successive trials in Experiments 1 (a) and 2 (b). Res. = response period, during which the stimulus would disappear when a response was made or when 1,000 ms had lapsed

effect of OA, $F(2.06,22.63$, with the Greenhouse-Geisser correction $)=258.47, p<.001, \eta_{\mathrm{p}}^{2}=.96$, showing the typical mental rotation effect (see Table 1).

We then assessed the influence of the preceding trial on the current trial. First, we examined the trials involving $0^{\circ}$ rotation. We expected that, because these trials would involve no rotation, they should show no direction repetition effect. As was expected, the mean RTs were similar for the repeated $0^{\circ}$ trials $(457.2 \mathrm{~ms})$ and the switched trials (449.1 ms), $t(11)=1.83, p=.10$ (two-tailed). For the remaining non- $0^{\circ}$ trials, we wanted to investigate whether the rotation direction of the preceding trial affected RT on the current trial and whether such an effect would vary by AOA, as we hypothesized. In addition, we also wanted to see whether the rotation direction repetition effect would depend on whether the consecutive stimuli were both normal or were the pairing of a mirror-reversed letter followed by a normal letter. (Please note that because of the use of a go/no-go paradigm, the pairings of normal/mirrorreversed letters and those of both mirror-reversed letters did not yield RT data.) Consequently, a 2 (rotation direction repetition: repeated, switched) $\times 2$ (AOA of the preceding stimulus: $\left.70^{\circ}, 140^{\circ}\right) \times 2$ (AOA of the current stimulus: $70^{\circ}$, $\left.140^{\circ}\right) \times 2$ (letter sequence: normal/normal vs. mirrorreversed/normal) repeated measures ANOVA was conducted on mean RTs for the current trial (averaged across clockwise and counterclockwise conditions).

Because we were interested in the effect of rotation repetition, we focused on its main effect and interactive 
effects with other variables. First, the four-way interaction was not significant, $F(1,11)=2.12, p=.17, \eta_{\mathrm{p}}^{2}=.16$. The three-way interaction involving rotation direction repetition, AOA of the current stimulus, and letter sequence was not significant as well, $F(1,11)=2.89, p=.12, \eta_{\mathrm{p}}^{2}=.21$. Nor was the three-way interaction concerning rotation direction repetition, AOA of the current stimulus, and AOA of the preceding stimulus significant, $F(1,11)=1.86, p=.20, \eta_{\mathrm{p}}^{2}=.15$.

In contrast, the two-way interaction between rotation direction repetition and AOA of the current stimulus was significant, $F(1,11)=13.50, p<.01, \eta_{\mathrm{p}}^{2}=.55$. Further simple effects analyses showed that the effect of rotation direction repetition was significant at both levels of AOA of the current stimulus, but it was much larger at the $140^{\circ}$ level than at the $70^{\circ}$ level of the current stimulus (see Fig. 2). Overall, the main effect of rotation repetition was also significant, $F(1,11)=71.92, p<.001, \eta_{\mathrm{p}}^{2}=.87$ (see Table 2 for more details).

It is worth noting that the results above based on RT were not driven by speed-accuracy trade-offs, because total error rates averaged across subjects were very low (about $2.2 \%$ ), and the error rates for $2 \times 2 \times 2 \times 2$ conditions were positively correlated with the RTs, $r=.48$ (see Fig. 2).
The foregoing results are consistent with our hypothesis that the repetition effect can occur without stimulus repetition. Mean RTs for the direction-repeated condition were shorter than those for the direction-switched condition. This difference was larger when more mental rotation was involved, indicating an alteration in the rate of mental rotation as a result of the AOA.

\section{Experiment 2}

One potential issue with Experiment 1 was that all six letters used as stimuli had the same natural orientation; that is, their openings faced the same direction (to the right). Natural orientation is essential to determining whether this stimulus is normal or mirror reflected (Hinton \& Parsons, 1981), so it is possible that the repetition effect found in Experiment 1 may have been due to this shared natural orientation in stimuli (i.e., stimulus similarity). To control for natural orientation of the stimuli, we used characters that have different natural orientations (i.e., facing different directions) in Experiment 2 to see whether the rotation repetition effect found in Experiment 1 would be replicated.

RTs (ms)

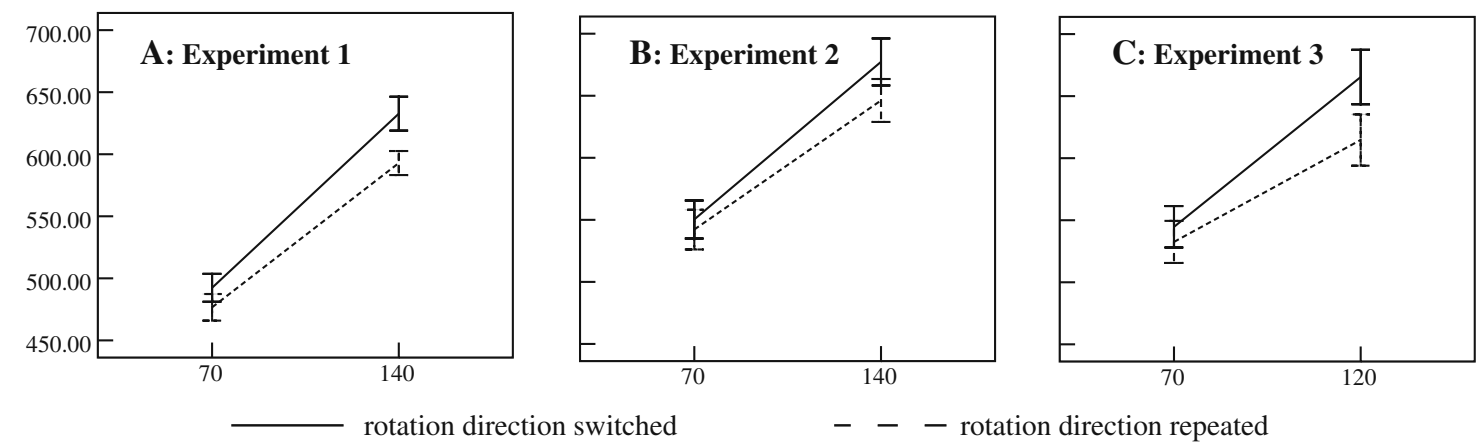

Error Rates
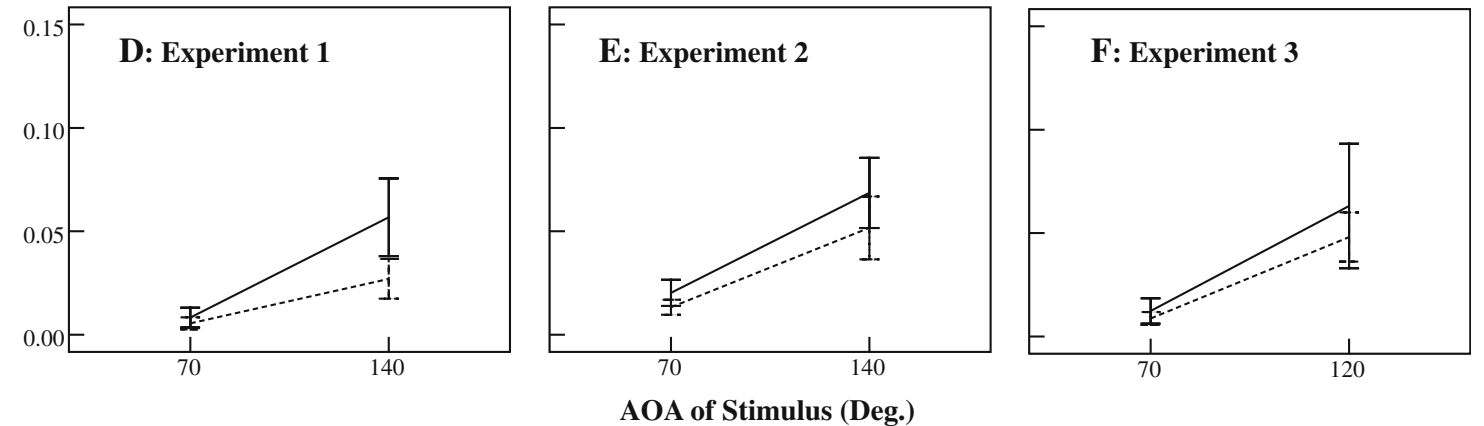

Fig. 2 The rotation direction repetition effect. Top panels show twoway interactions between rotation direction repetition and absolute orientation angle (AOA) of the current stimulus in mean response times (RTs). a Experiment 1. b Experiment 2. c Experiment 3. Bottom panels show two-way interactions between rotation direction repetition and AOA of the current stimulus in error rates. $\mathbf{d}$ Experiment 1. e Experiment 2. f Experiment 3. Error bars indicate the standard error of the mean for each condition 
Table $2 \mathrm{~F}$ values of other effects: Experiments 1 and 2

Note. AOA, absolute orientation angle

$* p<.05$

$* * p<.01$

\begin{tabular}{lll}
\hline Factors & \multicolumn{2}{l}{ Experiment } \\
\cline { 2 - 3 } & 1 & 2 \\
\hline AOA of the preceding stimulus & 3.94 & $7.43^{*}$ \\
AOA of the current stimulus & $689.35^{* *}$ & $133.40^{* *}$ \\
Letter sequence & $38.67^{* *}$ & .09 \\
Rotation-direction repetition $\times$ AOA of the preceding stimulus & 1.13 & 1.31 \\
Rotation-direction repetition $\times$ letter sequence & $6.63^{*}$ & $5.30^{*}$ \\
AOA of the preceding stimulus $\times$ AOA of the current stimulus & $16.59^{* *}$ & .26 \\
AOA of the preceding stimulus $\times$ letter sequence & $6.14^{*}$ & .32 \\
AOA of the current stimulus $\times$ letter sequence & $9.42^{*}$ & .14 \\
AOA of the preceding stimulus $\times$ AOA of the current stimulus $\times$ letter sequence & $13.93^{* *}$ & 4.07
\end{tabular}

\section{Method}

Twelve undergraduate students ( 2 male, 10 female) at Zhejiang University participated in this experiment. These students did not participate in Experiment 1. Each subject was paid 20 yuan. This experiment was approved by the Department of Psychology and Behavioral Sciences at Zhejiang University.

The design of this experiment was the same as that in Experiment 1, except for the characters used as stimuli and the rules for selecting them for each trial. We used four characters in this experiment. Two of them were Chinese characters (亡, 刃), and the other two were English characters $(\mathrm{G}, \mathrm{J})$. The openings of the characters $亡$ and $\mathrm{G}$ face right, whereas those of 刃 and $J$ face left. In this experiment, the current stimulus always faced a different direction than that on the preceding trial (see Fig. 1). Another minor difference was that the PC monitor used in this experiment was a 17-in. CRT with the resolution of $1,024 \times 768$ pixels.

Results and discussion

The mean RTs differed significantly across the five OAs of the current stimuli, $F(1.57,17.24)=114.35, p<.001, \eta_{\mathrm{p}}^{2}=.91$, indicating that mental rotation was involved (see Table 1). As in Experiment 1, the mean RTs for $0^{\circ}$ trials did not differ between the repeated trials $(494.7 \mathrm{~ms})$ and the switched trials (490.7 ms), $t(11)=0.58, p=.58$ (two-tailed). To examine the effect of the preceding trial on the current trial, we ran the same four-way repeated measures ANOVA as in Experiment 1. The results were very similar to those in Experiment 1. There was neither a four-way interaction, $F(1,11)=0.93$, $p=.36, \eta_{\mathrm{p}}^{2}=.08$, nor a three-way interaction among rotation direction repetition, AOA of the current stimulus, and letter sequence, $F(1,11)=0.16, p=.69, \eta_{\mathrm{p}}^{2}=.02$, nor was there a three-way interaction concerning rotation direction repetition, AOA of the current stimulus, and AOA of the preceding stimulus, $F(1,11)=0.11, p=.75, \eta_{\mathrm{p}}^{2}=.01$.
As in Experiment 1, the two-way interaction between rotation direction repetition and AOA of the current stimulus was significant, $F(1,11)=13.51, p<.01$, $\eta_{\mathrm{p}}^{2}=.55$. Further simple effects analyses also showed significant effects of direction repetition at both levels of AOA of the current stimulus, and it was larger at the $140^{\circ}$ level than at the $70^{\circ}$ level of the current stimulus (see Fig. 2). The main effect of rotation repetition was significant, $F(1,11)=50.12, p<.001, \eta_{\mathrm{p}}^{2}=.82($ see Table 2 for more details).

Again, the average error rates in all the conditions were low $(3.9 \%)$, and the error rates were positively correlated with the RTs, $r=.50$, indicating that the results based on RTs were not driven by speed-accuracy trade-offs (see Fig. 3).

These results replicated those of Experiment 1, indicating that the rotation repetition effect was robust and was not affected by characters' facing different directions on successive trials.

\section{Experiment 3}

Although Experiments 1 and 2 used different stimuli on successive trials and controlled for which direction the stimuli faced, they did not control for two other, somewhat related factors. First, although the group data showed the mental rotation effect and the rotation repetition effect, it was not guaranteed that all the subjects rotated all the trials as we wanted (i.e., using the shortest route to the upright position). As previous studies (Corballis \& McLaren, 1982; Heil, Bajrić, Rösler, \& Hennighausen 1997) have suggested, there might be a rotation aftereffect; namely, watching a rotating disk in one direction (e.g., clockwise) may increase the likelihood of rotating the following stimulus "the long way." To eliminate that possibility (or trials on which subjects rotated the long way), we needed to explicitly control for the direction of rotation. 

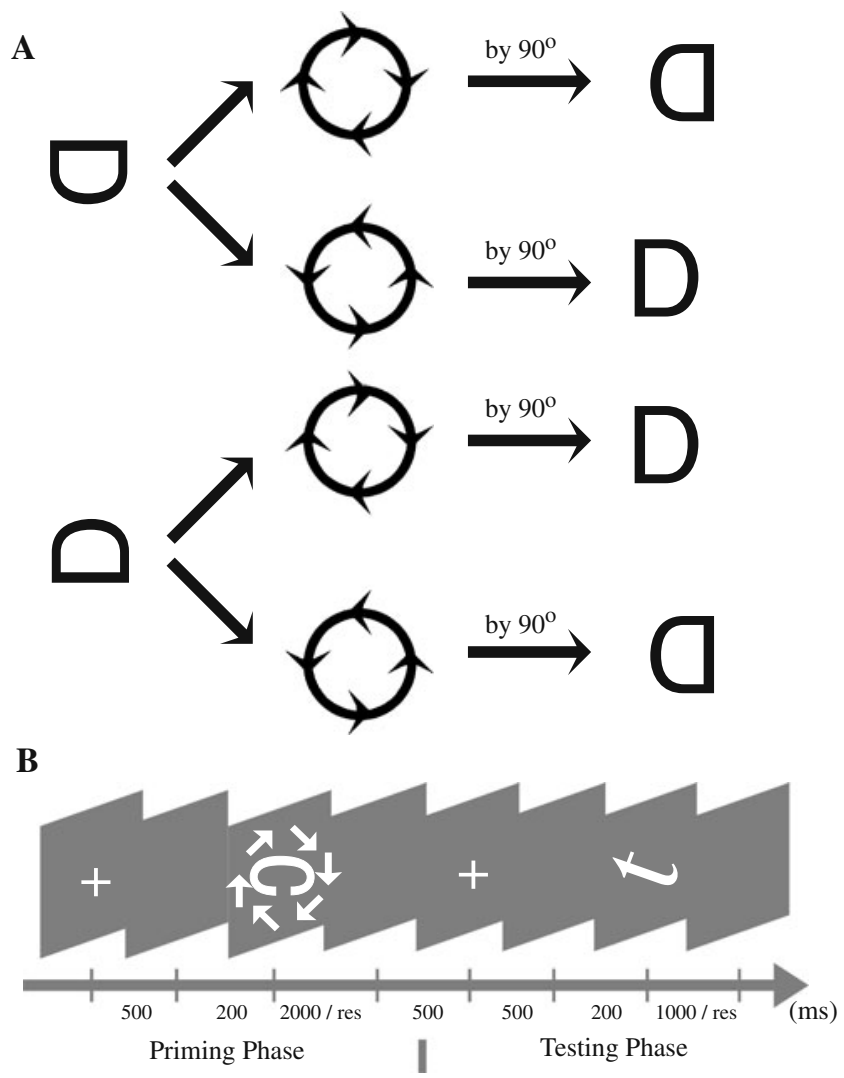

Fig. 3 Basic design of Experiment 3. a Illustration of how the priming phase (letter D as an example) was used to determine subjects' direction of mental rotation. $\mathbf{b}$ Schematic depictions of the time course of the two phrases of each trial. Res. = response period, during which the stimulus would disappear when a response was made or when a time limit was reached $(2,000 \mathrm{~ms}$ in the priming phase and $1,000 \mathrm{~ms}$ in the testing phase)

The second factor that was not considered in Experiments 1 and 2 was the internal reference frame effect (Graf, 2006; Graf, Kaping, \& Bülthoff 2005; Jolicœur, 1990; Robertson, Palmer, \& Gomez 1987). An internal reference frame can be regarded as a coordinate system for establishing position and orientation relative to the observer. It can be rotated to be aligned with the reference frame based on the stimulus. This adjusted internal reference frame can be reused for the subsequent stimulus with a similar orientation within a short time period. Consequently, there is a reduction in the mental rotation effect, similar to our results. Although in previous studies, the internal reference frame effect was restricted only to a brief interval between successive stimuli (e.g., less than $100 \mathrm{~ms}$ ) (Graf, Kaping, \& Bülthoff 2005; Robertson, Palmer, \& Gomez 1987) and the repeated response in the normal-backward discrimination task (Robertson, Palmer, \& Gomez 1987), our Experiments 1 and 2 could not rule out the possibility of an internal reference frame effect. In fact, in our Experiment 1, the interaction between AOA of the preceding stimulus and AOA of the current stimulus was significant (see Table 2), indicating that the mean RTs for the current trials were influenced by the relation between the orientations of the preceding stimulus and the current stimulus. After controlling for the natural orientations of the stimuli in Experiment 2, we no longer observed such an interaction.

To resolve these issues unambiguously, however, we had to have a better control of which way the subjects rotated the letters (e.g., not just assuming that they would rotate along the shortest route for the preceding stimulus and inferring its effect on the current stimulus). In Experiment 3 , we used three letters that were symmetrical along the midline $(\mathrm{C}, \mathrm{D}, \mathrm{K})$ and turned them $90^{\circ}$ either clockwise or counterclockwise and then primed the direction of their rotation to create either normal letters (by reversing the rotation) or mirror-reflected letters (by continuing the rotation for another $90^{\circ}$ ) (see Fig. 3a). Because the rotated letters depended on the direction of rotation, it was easy to ascertain whether the subjects followed the requested direction of rotation. The presentation of one of these letters would prime for a particular direction of mental rotation, and we then examined how this priming affected subsequent mental rotation performance with regular letters.

\section{Method}

Twelve undergraduate students ( 8 male, 4 female) at Zhejiang University participated in Experiment 3. These students did not participate in either Experiment 1 or 2 . These subjects received 20 yuan each for their participation. This experiment was approved by the Department of Psychology and Behavioral Sciences at Zhejiang University.

This experiment contained four blocks of 160 trials each. As in the other two experiments, the first block was treated as practice and, thus, was excluded from analysis. Six English characters were used as the stimuli: C, D, and K for the priming phase (see below), and $\mathrm{R}, \mathrm{F}$, and $\mathrm{t}$ for the testing phase. The reason for using lowercase $t$ was the following. Because all up-down symmetrical letters (except for $\mathrm{o}$ and $\mathrm{x}$, which are unfortunately right-left symmetrical as well) are capital letters, we could use those capital letters $(\mathrm{C}, \mathrm{D}$, and $\mathrm{K})$ only in the priming phase. For the testing phase, $\mathrm{R}, \mathrm{F}$, and $\mathrm{t}$ were chosen because they are the most different from $\mathrm{C}, \mathrm{D}$, and $\mathrm{K}$. Capital letters other than $\mathrm{R}$ and $\mathrm{F}$ either tend to share some visual structure with one of the priming letters (e.g., $\mathrm{C}$ and $\mathrm{G}, \mathrm{E}$ and $\mathrm{K}$ ) or were left-right symmetrical (e.g., A, O, T, W, U, V, X, Y), so lowercase t was selected (but its physical size was matched with $\mathrm{R}$ and $\mathrm{F}$ ).

Unlike in the other two experiments, a single trial in this experiment was divided into two phases: priming and testing (see Fig. 3b). In the priming phase, the stimulus was rotated to the horizontal (supine or prostrate) orientation from the canonical view of $\mathrm{C}, \mathrm{K}$, or D. Subjects were instructed to discriminate whether the stimulus was normal or mirror reflected if it was rotated, in the direction 
Table 3 Response time (RT) by orientation angle $(\mathrm{OA})$ in the testing phase in Experiment 3

\begin{tabular}{ll}
\hline OA & RT $(S E)$ \\
\hline $120^{\circ}$ & $636.81(22.87)$ \\
$70^{\circ}$ & $535.07(17.86)$ \\
$0^{\circ}$ & $500.89(15.24)$ \\
$+70^{\circ}$ & $538.59(15.56)$ \\
$+120^{\circ}$ & $642.63(19.52)$ \\
\hline
\end{tabular}

indicated by a circle of arrows, to the upright position. Arrows were needed because $\mathrm{C}, \mathrm{K}$, and $\mathrm{D}$ were purposely drawn to be perfectly symmetrical along the horizontal midline. As was mentioned earlier, relying on the sensitivity to rotation direction, we could determine whether subjects performed the rotation in the requested direction.

In the testing phase, a new stimulus ( $\mathrm{R}, \mathrm{F}$, or $\mathrm{t}$ ) was rotated and/or mirror reflected from its normal upright position, and subjects were asked to discriminate whether this stimulus was normal or mirror reflected by rotating it to the upright position in the direction in which the angular distance needed to be covered was less than $180^{\circ}$ (i.e., the shortest route).

This experiment had 40 conditions: 2 (rotation direction in the priming phase: clockwise, counterclockwise) $\times 2$ (character in the priming phase: normal or mirror reflected) $\times 2$ (character in the testing phase: normal or mirror reflected $) \times 5$ (OA of the stimulus in the testing phase: $\left.0^{\circ}, \pm 70^{\circ}, \pm 120^{\circ}\right)$. Each condition was randomly repeated 4 times in every block. We employed $\pm 120^{\circ}$, instead of $\pm 140^{\circ}$ as in Experiments 1 and 2, to accommodate the priming orientation (i.e., $90^{\circ}$ ), because $70^{\circ}$ and $140^{\circ}$ would have been too unbalanced in relation to $90^{\circ}$ (a difference of $20^{\circ}$ vs. $50^{\circ}$ ). It should also be noted that we used $70^{\circ}$, not $60^{\circ}$, in all three experiments in this study, because a pilot study showed that $70^{\circ}$, not $60^{\circ}$, resulted in a rotation effect adequate for studying the repetition effect.

In both phases of a trial, a fixation was presented for $500 \mathrm{~ms}$ at the beginning, followed by a 200 -ms blank and then the stimulus. The stimulus stayed on the screen until the subject had responded or the time limit $(2,000 \mathrm{~ms}$ in the priming phase, $1,000 \mathrm{~ms}$ in the testing phase) was reached. There was a 500-ms blank after both phases. Subjects were asked to press "B" only when seeing a normal character, and not to respond when seeing a mirror-reflected character. Both speed and accuracy were emphasized (see Fig. 1). Because different characters were used in the priming and the testing phases, there would be no stimulus repetition.

\section{Results and discussion}

A one-way repeated measures ANOVA showed that the mean RTs for correct responses differed significantly across the five OAs in the testing phase, $F(1.92,21.09)=97.20, p<.001$, $\eta_{\mathrm{p}}^{2}=.90$, showing a strong mental rotation effect (see Table 3). We then conducted a 2 (rotation direction repetition: repeated vs. switched) $\times 2$ (AOA of current stimulus: $70^{\circ}$ vs. $\left.120^{\circ}\right) \times 2$ (letter sequence: normal/normal vs. mirror-reversed/ normal) repeated measures ANOVA on mean RTs for correct trials in the testing phase. In order to examine the priming effect, we further limited the data to those trials on which the subjects also performed correctly in the priming phase.

The three-way interaction was not significant, $F(1,11)=$ 2.44, $p=.15$. The two-way interaction between rotation direction repetition and AOA of the current stimulus was significant, $F(1,11)=24.74, p<.01, \eta_{\mathrm{p}}^{2}=.69$. Further simple effects analyses showed that the effect of rotation direction repetition was significant at both levels of AOA of the current stimulus, but it was much larger at the $120^{\circ}$ level than at the $70^{\circ}$ level of AOA of the current stimulus (see Fig. 2). The main effect of rotation repetition was significant, $F(1,11)=$ $71.82, p<.001, \eta_{\mathrm{p}}^{2}=.87$ (see Table 4 for more details).

Total error rates averaged across subjects were again very low $(2.2 \%$ for the priming phase and $1.9 \%$ for the testing phase). The error rates for the $2 \times 2 \times 2$ conditions analyzed above were positively correlated with the RTs, $r=.60$, indicating no speed-accuracy trade-offs (see Fig. 2).

The results of Experiment 3 were similar to those of the other two experiments. In this experiment, the stimuli for the priming phase had no certain orientation. As a result, there should be no orientation similarity between letters for priming, ruling out the possibility that the rotation repetition effect was caused by perceptual priming based on orientation similarity. We also eliminated those trials in which subjects did not rotate the priming letter in the direction requested. This experiment also showed that, after controlling for the rotation direction (as well as natural orientation) of the priming stimuli to minimize the reference frame effect, we still found the rotation-direction repetition effect. These results provided strong evidence that the repetition effect of different stimuli was due to increased efficiency of mental rotation processing that was activated on the preceding trial.

\section{General discussion}

The results of the present study showed that mental rotation of the current stimulus was facilitated when the preceding

Table $4 \mathrm{~F}$ values of other effects in Experiment 3

\begin{tabular}{ll}
\hline Factors & $F$ values \\
\hline AOA of test stimulus & $128.92^{* *}$ \\
Letter sequence & 2.02 \\
Rotation direction repetition $\times$ letter sequence & $15.25^{*}$ \\
AOA of test stimulus $\times$ letter sequence & 1.17 \\
\hline
\end{tabular}

Note. AOA, absolute orientation angle.

$* p<.05$

$* * p<.01$ 
stimulus showed rotation in the same direction. In the following paragraphs, we discuss our findings in the context of previous literature on related concepts. First, this study expanded previous findings of a simple repetition-of-stimulus effect (i.e., when the same stimulus was repeated). We found that the rotation direction repetition effect across different stimuli was evident even after natural orientations of the letters were controlled for. This finding suggests that the rotation direction repetition effect was due not to perceptual processing (or stimulus encoding) or response selection but, rather, to increased efficiency in mental rotation per se.

Second, our rotation direction repetition effect is different from the cuing effect. The cuing effect means any potential effect on mental rotation as a result of cuing which way the forthcoming stimulus would face (Cooper \& Shepard, 1973). On the surface, the cuing effect and the rotation direction repetition effect appear similar. Indeed, if cuing had led to reduction in mental rotation, it would have been difficult to distinguish them. However, Cooper and Shepard did not find the cuing effect; that is, simply indicating the orientation of the forthcoming stimulus did not reduce the RT. The main difference between cuing and rotation direction repetition is that cuing per se may not involve a mental rotation process. Only an actual trial of rotation would affect the following rotation process. The results of our Experiment 3 are consistent with this argument. In that experiment, we found that after we controlled for orientation and direction of rotation (i.e., no cuing), we still found the direction repetition effect. On the basis of these results, we can infer that cuing probably does not involve mental rotation. This particular conjuncture, of course, needs to be further tested in future research.

Third, our repetition effect was not due to the reference frame effect. In Experiment 1, we found a significant interaction between angle of the preceding stimulus and angle of the current stimulus. Because the stimuli in this experiment all faced the same way, there might have been an effect of a repeated reference frame. After controlling for the orientation of the stimuli (Experiment 2) and the rotation direction (Experiment 3) to eliminate or at least minimize the reference frame effect, the rotation-direction repetition effect was still evident. It is also worth noting that evidence for the reference frame effect came mainly from recognition tasks, not from mental rotation tasks. When Robertson, Palmer, and Gomez (1987) found the reference frame effect in a mental rotation task, it was limited to the same-reflection condition, which can be explained by category repetition (normal or mirror reversed). The results from a recent study further showed that the reference frame effect found in recognition tasks may be based on a consolidation process of decision making (Dux \& Harris, 2007). Taken together, the reference frame effect does not seem to play a role in the rotation direction repetition effect.

Fourth, Kung and Hamm (2010) recently found that the RT function of their mental rotation task for alphanumeric characters was not linear. They believed that when the stimulus was closer to the upright, the probability of involving mental rotation was lower and, therefore, the RT function was flatter. They assumed that the observed RT at each AOA was a weighted average of RT from trials with mental rotation and that from trials without mental rotation, and the ratio of trials involving mental rotation was given by an empirical formula (i.e., $r=\mathrm{AOA} / 180$ ). With these assumptions, RT at each AOA when all the trials would involve mental rotation could be estimated. They showed that the estimated (or transformed) RT data were linear, as they expected. Would the hypothesized differential proportions of rotated trials for each AOA explain our results? To address this question, we conducted a post hoc analysis. Using the empirical formula suggested by Kung and Hamm, we transformed the RT data for the two conditions (see Fig. 4).
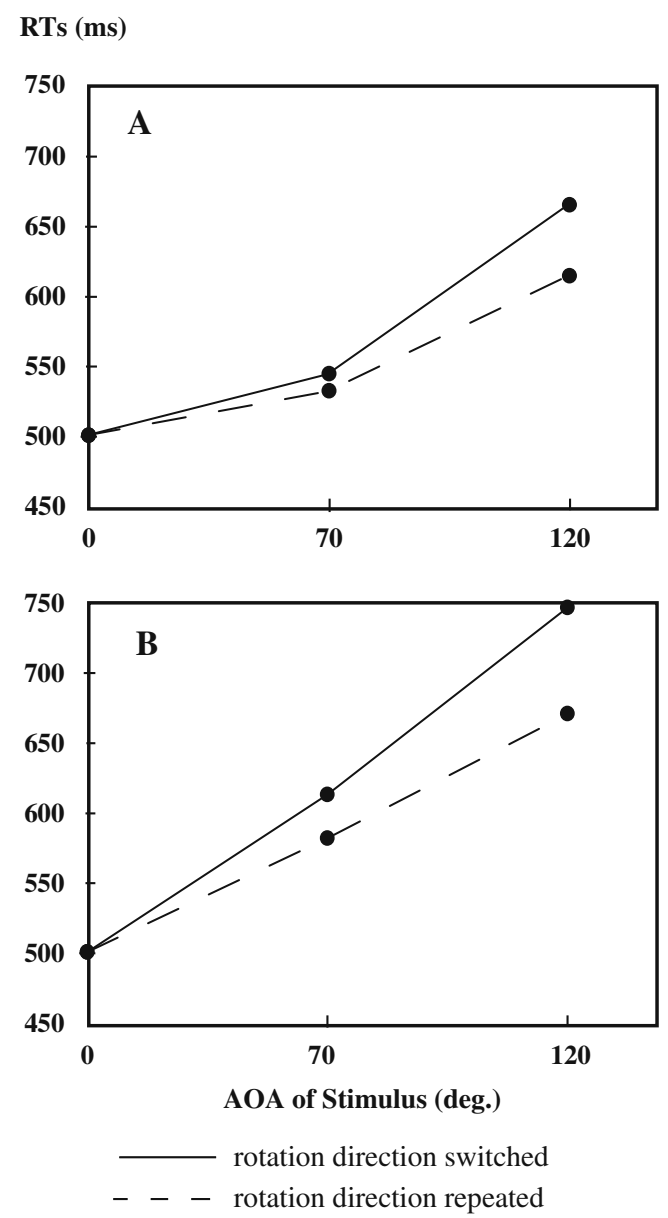

Fig. 4 Results from applying Kung and Hamm's (2010) formula to the data from the two conditions in Experiment 3. a Response time (RT) functions for the original data. b RT functions for the estimated/ transformed data 
Results showed that the transformed RT data were linear for both conditions - namely, no differential ratio changes for the two conditions. These results, together with a lack of the repetition effect for the $0^{\circ}$ trials, provided strong evidence that the rotation direction repetition effect was due to increased efficiency of mental rotation.

In sum, after carefully controlling for natural orientations and rotation directions, our experiments showed that mental rotation of the current stimulus was facilitated when the preceding stimulus showed rotation of the same direction. This rotation direction repetition effect was independent of stimulus repetition or related perceptual processing but, rather, was due to increased efficiency in the mental rotation process per se. Future research should aim to understand the cognitive and neural mechanisms (e.g., a process akin to precise reinstatement of neural patterns; Xue et al., 2010) involved in this increased efficiency.

Acknowledgements This research was supported by NSF of China, Grant 30470573.

Open Access This article is distributed under the terms of the Creative Commons Attribution Noncommercial License which permits any noncommercial use, distribution, and reproduction in any medium, provided the original author(s) and source are credited.

\section{References}

Altmann, E. M., \& Gray, W. D. (2008). An integrated model of cognitive control in task switching. Psychological Review, 115, 602-639.

Cooper, L. A., \& Shepard, R. N. (1973). Time required to prepare for a rotated stimulus. Memory \& Cognition, 1, 246-250.

Corballis, M. C., \& McLaren, R. (1982). Interaction between perceived and imagined rotation. Journal of Experimental Psychology: Human Perception and Performance, 8, 215-224.

Dobbins, I. G., Schnyer, D. M., Verfaellie, M., \& Schacter, D. L. (2004). Cortical activity reductions during repetition priming can result from rapid response learning. Nature, 428, 316-319.

Dux, P., \& Harris, I. (2007). Viewpoint costs occur during consolidation: Evidence from the attentional blink. Cognition, 104, 47-58.

Graf, M. (2006). Coordinate transformations in object recognition. Psychological Bulletin, 132, 920-945.

Graf, M., Kaping, D., \& Bülthoff, H. H. (2005). Orientation congruency effects for familiar objects: Coordinate transformations in object recognition. Psychological Science, 16, 214-221.

Heil, M., Bajrić, J., Rösler, F., \& Hennighausen, E. (1997). A rotation aftereffect changes both the speed and the preferred direction of mental rotation. Journal of Experimental Psychology: Human Perception and Performance, 23, 681-692.

Heil, M., Rauch, M., \& Hennighausen, E. (1998). Response preparation begins before mental rotation is finished: Evidence from envent related brain potentials. Acta Psychologica, 99, 217-232.
Hinton, G. E., \& Parsons, L. M. (1981). Frames of reference and mental imagery. In J. Long \& A. Baddeley (Eds.), Attention and performance IX (pp. 261-277). Hillsdale: Erlbaum.

Ilan, A. B., \& Miller, J. (1994). A violation of pure insertion: mental rotation and choice reaction time. Journal of Experimental Psychology: Human Perception and Performance, 20, 520-536.

Jolicœur, P. (1990). Orientation congruency effects on the identification of disoriented shapes. Journal of Experimental Psychology: Human Perception and Performance, 16, 351-364.

Koriat, A., \& Norman, J. (1984). What is rotated in mental rotation? Journal of Experimental Psychology. Learning, Memory, and Cognition, 10, 421-434.

Koriat, A., \& Norman, J. (1988). Frames and images: sequential effects in mental rotation. Journal of Experimental Psychology. Learning, Memory, and Cognition, 14, 93-111.

Koriat, A., \& Norman, J. (1989). Establishing global and local correspondence between successive stimuli: The holistic nature of backward alignment. Journal of Experimental Psychology. Learning, Memory, and Cognition, 15, 480-494.

Kung, E., \& Hamm, J. (2010). Model of rotated mirror/normal letter discriminations. Memory \& Cognition, 38, 206-220.

Mayr, U., \& Kliegl, R. (2003). Differential effects of cue changes and task changes on task-set selection costs. Journal of Experimental Psychology. Learning, Memory, and Cognition, 29, 362-372.

Meiran, N. (2000). Modeling cognitive control in task-switching. Psychological Research, 63, 234-249.

Meiran, N., \& Gotler, A. (2001). Modelling cognitive control in task switching and ageing. European Journal of Cognitive Psychology, 13, 165-186.

Monsell, S. (2003). Task switching. Trends in Cognitive Sciences, 7, 134-140.

Monsell, S., Yeung, N., \& Azuma, R. (2000). Reconfiguration of taskset: Is it easier to switch to the weaker task? Psychological Research, 63, 250-264.

Pashler, H., \& Baylis, G. (1991). Procedural learning: II. Intertrial repetition effects in speeded-choice tasks. Journal of Experimental Psychology: Human Learning and Memory, 17, 33-48.

Philipp, A. M., Jolicœur, P., Falkenstein, M., \& Koch, I. (2007). Response selection and response execution in task switching: Evidence from a go-signal paradigm. Journal of Experimental Psychology. Learning, Memory, and Cognition, 33, 1062-1075.

Robertson, L. C., Palmer, S. E., \& Gomez, L. M. (1987). Reference frames in mental rotation. Journal of Experimental Psychology. Learning, Memory, and Cognition, 13, 368-379.

Schacter, D. L., Chiu, C. Y. P., \& Ochsner, K. N. (1993). Implicit memory: A selective review. Annual Review of Neuroscience, 16, 159-182.

Steinhauser, M., \& Hübner, R. (2006). Response-based strengthening in task shifting: Evidence from shift effects produced by errors. Journal of Experimental Psychology: Human Perception and Performance, 32, 517-534.

Wexler, M., Kosslyn, S. M., \& Berthoz, A. (1998). Motor processes in mental rotation. Cognition, 68, 77-94.

Wiggs, C. L., \& Martin, A. (1998). Properties and mechanisms of perceptual priming. Current Opinion in Neurobiology, 8, 227-233.

Wohlschläger, A., \& Wohlschläger, A. (1998). Mental and manual rotation. Journal of Experimental Psychology: Human Perception and Performance, 24, 397-412.

Xue, G., Dong, Q., Chen, C., Lu, Z., Mumford, J., \& Poldrack, R. (2010). Greater neural pattern similarity across repetitions is associated with better memory. Science, 330, 97-101. 